\title{
Guia norteador para condução de grupo focal na identificação de competências gerenciais: Relato de experiência
}

\footnotetext{
Laura Andrian Leal ${ }^{1}$ e Silvia Helena Henriques ${ }^{2}$

1 Enfermeira, Doutoranda da Escola de Enfermagem de Ribeirão Preto, Universidade de São Paulo, Brasil | laura.andrian.leal@usp.br | https://orcid.org/0000-0002-8563-8980

2 Professora Associada do Departamento de Enfermagem Geral e Especializada da , Escola de Enfermagem de Ribeirão Preto, Universidade de São Paulo, Brasil shcamelo@eerp.usp.br | http://orcid.org/0000-0003-2089-3304

Resumo: Introdução: A utilização da técnica de Grupos Focais na enfermagem estão presentes na literatura, entretanto, ressaltam-se lacunas com relação a guias ou roteiros que podem nortear sua condução. Objetivos: Apresentar a experiência dos pesquisadores na elaboração de um guia para nortear a condução de grupos focais relacionados a identificação de competências gerenciais prioritárias ao desenvolvimento de discentes de enfermagem. Métodos: Relato de experiência realizado em uma universidade brasileira. O guia foi construído tendo por base artigos disponíveis da literatura, referencial proposto e a experiência das autoras em um curso da Espanha. Este guia foi validado e testado por quatro especialistas de gestão em enfermagem, nível doutorado. Após construção e validação, foram realizados três grupos focais com docentes da área de gerenciamento da IES selecionada. A coleta ocorreu no período de março a abril de 2020 e foi realizada análise temática indutiva. Resultados: A construção de um guia norteador demonstrou ser estratégia capaz de identificar aspectos relevantes para a formação do enfermeiro no que diz respeito a identificação das competências gerenciais. Essa construção pôde servir de base para condução de demais grupos. Ademais, a partir do guia construído e sua implementação identificou-se competências gerenciais prioritárias, tais como: liderança, comunicação, relacionamento interpessoal e tomada de decisão gerencial. Conclusões: A estratégia de grupos focais com docentes de enfermagem acompanhadas por um guia norteador demonstrou ser positiva na identificação de competências gerenciais prioritárias ao ensino de enfermagem, favorecendo a elaboração de estratégias para o desenvolvimento destas competências e consequentemente contribuir para a inserção deste profissional no mercado de trabalho.
}

Palavras-chave: Grupos Focais; Competência Profissional; Pesquisa Qualitativa; Enfermagem.

\section{Guiding Guide for Conducting a Focus Group in Identifying Managerial Skills: Experience Report}

Abstract: Introduction: The use of the Focus Groups technique in nursing is present in the literature, however, gaps are highlighted in relation to guides or scripts that can quide their conduct in the area of identifying managerial competences. Objectives: To present the researchers' experience with the elaboration of a guide to guide the conduct of FG related to the identification of management skills that are priority to the development of nursing students. Methods: Experience report carried out in a Brazilian Public Higher Education Institution. The guide was built based on articles available in the literature, reference proposed and the experience of the authors in a course offered by Spain. This guide has been validated and tested by four $\mathrm{PhD}$ specialists. After construction and validation, three focus groups were held with professors from the management area of the selected institution, who had worked for more than two years in the function. The collection took place from March to April 2020. The groups were had an average duration of 55 minutes. An inductive thematic analysis was performed. Results: The construction of a guiding guide for driving must follow rigorous steps evidenced by the referential, and proved to be a strategy capable of identifying relevan aspects for the training of nurses with regard to the identification of managerial skills. Also can serve as a basis for conducting other groups. In addition, from the guide built and its implementation, priority managerial competencies were identified: leadership, communication, interpersonal relationships and managerial decision making. Conclusions: The strategy of focus groups with nursing professors accompanied by a guiding guide proved to be positive in identifying managerial competencies that are a priority in nursing education, enabling the formation of a better prepared professional to face the job market.

Keywords: Focus Groups; Professional Competence; Qualitative Research; Nursing. 


\section{Introdução}

Este artigo é parte da tese intitulada "Competências gerenciais para discentes de enfermagem: implementando metodologia ativa de ensino" que será apresentada futuramente na Universidade de São Paulo. O referido estudo teve como um dos objetivos a identificação das competências gerenciais prioritárias ao desenvolvimento do discente de enfermagem para atuação hospitalar e, para esta identificação foi necessária ampla discussão entre os docentes da área gerencial, o que ocorreu por meio da utilização da técnica de Grupo Focal (GF).

As competências gerenciais estão relacionadas aos conhecimentos, habilidades e atitudes da área de gestão, abrangendo liderança; tomada de decisão; comunicação; relacionamento interpessoal, custos, entre outros (Ferracioli et al., 2020).

O GF pode se constituir como método efetivo para discussões neste âmbito, podendo contribuir para o preparo e formação do profissional. Além disso, tem sido aplicado como técnica com objetivo de coletar informações sobre determinado tema específico por meio da discussão participativa entre os seus integrantes, reunidos em um mesmo local e durante certo período de tempo. Isso proporciona a troca de experiências, conceitos e opiniões entre os participantes. Origina discussões e elabora táticas grupais para solucionar problemas e transformar realidades, pautando-se na aprendizagem e na troca de experiências sobre a questão em estudo (Dall'agnol et al., 2012).

$\mathrm{Na}$ enfermagem a realização desta técnica já vem sendo descrita em outros relatos de experiências, como técnica exitosa, entretanto, não é tarefa simples, uma vez que exige dos pesquisadores atitudes adequadas para o aprofundamento acerca dos significados e subjetividade dos participantes a respeito dos aspectos que envolvem seu processo de trabalho (Soares et al., 2016; Santos et al., 2019).

Para realização dos grupos é necessário dispor a condução de algumas etapas, como: 0 período de sensibilização dos participantes, desenvolvimento do GF com organização do ambiente e condução do grupo propriamente dita. Destaca-se que para concretização destas etapas é preciso dispor de um temário ou guia, que serve como um auxílio para a memorização de questões importantes a serem tratadas e permite melhor compreensão dos objetivos de pesquisa para o GF (Gatti, 2012).

Contudo, a este respeito, cabe destacar a baixa produção de estudos na literatura que focalizam guias para auxiliar o desenvolvimento de GF. Para tanto, realizou-se uma consulta na literatura a fim de aprofundar na compreensão sobre a temática nas bases de dados eletrônicas, Medical Literature Analysis and Retrieval System Online - MEDLINE, Literatura Latino-Americana e do Caribe em Ciências da Saúde-LILACS, e Web of Science, utilizando os descritores em ciências da saúde (DeCS): Guia; Grupos Focais, Pesquisa Qualitativa e Competência Profissional; e com Medical Subject Headings (MeSH): Guideline; Focus Groups; Qualitative Research; e Professional Competence; combinados com o operador booleano AND. Foram considerados artigos disponíveis de forma completa, publicados nos idiomas português, inglês e espanhol, no período de 2000 a 2019. A busca constatou a carência de produção atualizada acerca de guias para nortear a realização de GF.

Foi utilizado como questão norteadora: A elaboração de um guia ou roteiro pode auxiliar a condução de um GF na identificação de competências gerenciais prioritárias ao desenvolvimento de discentes de enfermagem?

Acredita-se na aplicabilidade dos grupos focais em pesquisas qualitativas, e assim faz-se necessário aprofundar no conhecimento de pesquisas que utilizam métodos e/ou estratégias que possam auxiliar na sua condução, com consequências positivas para investigações na área da saúde.

Deste modo, este artigo teve o objetivo de apresentar a experiência dos pesquisadores na elaboração de um guia para nortear a condução de GF relacionado a identificação de competências gerenciais prioritárias ao desenvolvimento de discentes de enfermagem. 


\section{Metodologia}

Trata-se de um relato de experiência sobre a construção de um guia norteador para condução de GF. Foi realizado em uma Instituição de Ensino Superior (IES) pública brasileira do interior paulista. A fim de nortear as discussões nos grupos focais, inicialmente foi construído um guia que teve como base uma consulta na literatura realizada de setembro a outubro de 2019, o referencial proposto por Gatti (2012) e acresce-se a isso a experiência das autoras na implementação do método. A construção deste guia também foi pautada em um curso intitulado "Cómo realizar grupos focales", disponibilizado pela FUNDACION INDEX, Espanha, de modo virtual, onde uma das autoras participou. Com relação a consulta na literatura inicialmente foram selecionadas oito publicações potenciais, que após a exclusão dos materiais que não respondiam à questão norteadora da introdução estudo, o resultado ficou constituído por duas publicações, as de Kinalski et al. (2017) e Gui (2003).

O guia foi validado e testado por quatro especialistas na área de gestão de enfermagem com nível doutorado e experiência em estudos qualitativos. Após esta etapa, foram realizados três $\mathrm{GF}(\mathrm{s})$ com os docentes do ultimo ano do ensino de graduação em enfermagem da referida instituição. Com relação aos critérios de seleção dos participantes, foram convidados docentes responsáveis por disciplinas da área de gerenciamento de enfermagem hospitalar. A escolha se deu pela temática a ser investigada. Foram selecionados os docentes que tinham mais de dois anos de experiência (por considerar que este profissional tinha maior experiência educacional e prática), totalizando 23 docentes. Os docentes afastados ou de licença-saúde foram excluídos da pesquisa.

A implementação dos grupos ocorreu no período de março a abril de 2020. Para facilitar a compreensão da realização do GF, utilizou-se o referencial proposto por Gatti (2012) e optou-se por organizá-lo conforme as etapas propostas na sequência:

1ํ passo: Período de sensibilização, seleção e montagem dos GF

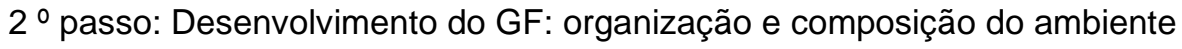

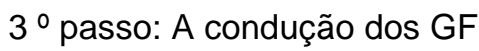

\subsection{Período de Sensibilização, Seleção e Montagem dos GFs}

$\mathrm{Na}$ fase de seleção dos participantes para compor o GF, foram convidados de 4 a 10 docentes. A constituição do GF é feita de forma intencional e pretende-se que haja, pelo menos, um ponto de semelhança entre os participantes (Gatti, 2012). Assim, os GF(s) foram realizados em consonância com os períodos de trabalho e estudo dos docentes, ou seja, período da manhã e tarde, bem como de acordo com a disponibilidade de cada um para os encontros. Para registro das informações utilizou-se três gravadores digitais durante as sessões, com o intuito de registrar na íntegra os discursos; além disso, todas as observações e impressões foram registradas em um diário de campo.

\subsection{Desenvolvimento do Grupo Focal: Organização e Composição do Ambiente}

A escolha do local de realização das sessões do GF tem fundamental importância na adesão dos participantes; é preciso estabelecer um ambiente propício às interações. Diante disso, os participantes foram distribuídos em torno de uma mesa oval em salas próprias da IES selecionada. Ademais, os grupos foram realizados pelo pesquisador (moderador) e um auxiliar de pesquisa (observador) visando descrever as situações identificadas. O papel primordial do observador é facilitar a discussão com o moderador após o encontro com o GF, evitando conclusões precipitadas por parte do moderador, acerca de suas impressões e registros (Gatti, 2012). 
Ao moderador do trabalho cabe o importante papel de manter-se atento às discussões, intervindo sempre que há necessidade de esclarecimento das ideias (Dall'agnol et al., 2012).

\subsection{A Condução dos GF}

Todas as atividades tiveram tempo definido. Assim, foi realizado o encontro com os grupos para a discussão sobre o tema pesquisado. O moderador e o observador receberam cada participante de maneira cordial e confeccionaram de um crachá para melhor identificá-lo. O desenvolvimento do GF foi orientado pelas questões norteadoras do guia. Ademais, ressalta-se que o tempo de duração e o número de participantes de cada reunião grupal podem variar, mas não deve ultrapassar 1 hora, o que aconteceu em nossa investigação. No encerramento dos grupos dessa experiência, o coordenador realizou a síntese do trabalho do grupo. Assim os GFs tiveram duração média total de 55 minutos.

Após a construção e execução dos GF(s) com os docentes realizou-se a transcrição das gravações e foram acrescidas às anotações e reflexões do moderador e do observador. Utilizou-se a análise temática indutiva para interpretação dos dados provenientes das discussões do GF. Este tipo de análise é um método que identifica, analisa e relata os padrões (temas) dentro dos dados; organiza e descreve seu conjunto de dados em detalhes que por meio da sua liberdade teórica (Braun \& Clarke, 2006).

Por se tratar de uma pesquisa desenvolvida com seres humanos, foi respeitado as determinações da Resolução de oㅜ 466/2012 e os participantes assinaram o Termo de Consentimento Livre e Esclarecido (TCLE). Foi submetido e aprovado pelo Comitê de Ética e Pesquisa da Instituição proponente, sob aprovação CAAE número 21390619.1.0000.5393.

\section{Resultados}

Apresentam-se aqui os resultados da experiência em três capitulos. A princípio pretendese mostrar o guia construído e validado para condução dos grupos focais, apresentar a realização dos GF(s) à partir do guia construído e finalmente as competências identificadas por meio destes grupos.

\subsection{Guia Norteador para Condução}

O guia construído para condução dos grupos focais seguiu as fases elencadas previamente no método, conforme nosso referencial teórico, evidenciado no Quadro 1:

Quadro 1. Guia norteador para GF com base na identificação das competências gerenciais prioritárias ao ensino do aluno de enfermagem. Brasil, 2021.

GUIA NORTEADOR GRUPO FOCAL:

Considera-se que o GF estará guiado pelos seguintes passos: 1 ํ Período de sensibilização, seleção e montagem dos GF (onde será feito a seleção e convite aos docentes); 2ํo passo: Desenvolvimento do GF: organização e composição do ambiente (onde o ambiente e espaço físico serão organizados tendo preferência a um ambiente limpo, organizado e prazeroso. Dispor de gravadores digitais sobre a mesa e seguir as etapas de organização: preparação,

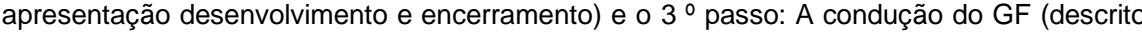
abaixo):

\section{A CONDUÇÃO DO GF}

Início: Preparação (duração $05 \mathrm{~min}$ ):

- $\quad$ Receber os participantes de maneira cordial e solícita;

- $\quad$ Confecção do crachá de identificacão:

- $\quad$ Acomodar os participantes (em uma disposicão de círculo):

- $\quad$ Observador e moderador entregam o TCLE e aguardam assinatura;

- $\quad$ Entrega do questionário sociodemográfico aos participantes para autopreenchimento; 
Apresentação (duração $10 \mathrm{~min}$ )

- $\quad$ Apresentação do coordenador (moderador) e observador:

Bom dia!/Boa tarde! Meu nome é ...... sou coordenadora da atividade e tenho o propósito de apresentar e estimular a participação e condução do grupo. Agradeço a presença de todos aqui reunidos, será um período para nos conhecermos melhor e trocar várias experiências.

Após o observador explica sua função. Meu nome é.......e durante o encontro vou acompanhando tudo que vai acontecendo e registrando anotações e as pessoas que quiserem falar, podem levantar a mão, pois assim ajudando organizar para que todos possam participar.

- Será feita uma breve introducão da temática com objetivo de situar o grupo no desenvolvimento das atividades: Considerando que no processo de formação de enfermeiros, segundo as Diretrizes Curriculares Nacionais (DCN), as Instituições de Ensino Superior (IES) devem promover o desenvolvimento das seguintes competências: Atenção á saúde; Tomada de decisão; Comunicação; Liderança; Administração e gerenciamento e Educação Permanente (BRASIL, 2001); queremos que vocês compartilhem suas experiências como docente refletindo algumas questões que iremos apresentar.

- $\quad$ Assegurar que não existem opiniões corretas e que opiniões contrárias são bem-vindas; e não existe uma ordem e/ou prioridade para início da participação de cada membro, deixandoos à vontade;

- $\quad$ Moderador faz alguns acordos: Consenso entre horário de início e término; Solicitando permissão para gravação. (Se todos concordarem, iniciar a gravação neste momento); Quando algum participante não quiser mais participar avisar à observadora; Acordo para que os participantes no desenvolvimento falem um de cada vez; Solicitar para que antes de cada um expor a sua opinião para dizer seu nome antes para poder identificar com maior clareza na gravação; Acordo do sigilo compartilhado sobre fatos que aconteceram ou coisas que outras pessoas falarem.

- $\quad$ Promover a apresentação dos participantes entre si.

\section{Desenvolvimento (duração 30-40 $\mathrm{min}$ ):}

- $\quad$ Moderador inicia: Bom pessoal, então de acordo com as explicações anteriores iniciaremos as discussões. (Reforçar a questão do tempo máximo de discussão).

- Propor questões norteadoras para o debate:

1- $\quad$ Em se tratando de competências gerenciais para área hospitalar, quais vocês consideram emergentes para o ensino do graduando de enfermagem? (Pelo menos duas competências prioritárias que possam ser incorporadas no ensino e desenvolvidas pelo discente de enfermagem, ainda no contexto da graduação para atuação no hospital).

2- $\quad$ As competências podem ser desenvolvidas de diversas formas, ou seja, o docente pode utilizar estratégias de ensino que aprimorem conhecimentos, habilidades e atitudes dos graduandos. Pensando nisso, quais estratégias têm sido desenvolvidas para implementar as competências gerenciais aos graduandos e/ou que vocês consideram relevantes para o ensino na graduação dos enfermeiros?

Neste momento observador irá registrar os depoimentos advindos das discussões.

- $\quad$ Fazer uma breve pausa $(05 \mathrm{~min})$ para observador e moderador sintetizarem momentos anteriores. Enquanto isso é servido um café aos participantes.

\section{Encerramento (duração: $10 \mathrm{~min}$ )}

- $\quad$ Retomar e informar a síntese da discussão realizada pelo grupo: O objetivo é identificar temas principais das discussões. Deve-se conceder espaço para participantes manifestarem quando a veracidade da síntese ou para esclarecimentos se necessário.

- $\quad$ Agradecer novamente a participação.

- $\quad$ Encerrar a sessão.

Momento posterior ao término do grupo: nesse momento moderador e observador irão avaliar se a condução das discussões foi adequada e se as necessidades de informações foram satisfeitas, ou se o tema precisa ser revisto e se será necessário mais grupos.

\subsection{Realização dos GF}

Dos 23 docentes convidados, efetivamente participaram 12 (52,17\%); 10 (43,47\%) recusaram por questões de indisponibilidade de horário entre os encontros e um $(4,34 \%)$ por não concordância em participar da pesquisa, sem um motivo aparente. 
Assim, foram realizados três $\mathrm{GF}(\mathrm{s})$, sendo quatro participantes em cada. Ainda, para realização dos mesmos, ressalta-se que foi solicitada a limpeza prévia dos ambientes e atenderam-se às necessidades de ventilação e de iluminação. Atentamos particularmente em garantir não só a privacidade dos envolvidos, mas também propiciar-Ihes ambiente aconchegante, incluindo servir-lhes água mineral, música ambiente e guloseimas. A cordialidade no acolhimento dos participantes desde sua chegada ao local foi uma preocupação constante. Essas características permitiram acesso facilitado aos participantes por estarem localizadas na própria instituição

Ademais, o coordenador foi representado pela primeira pesquisadora, sendo que esta coordenou os grupos com o guia e a observadora foi representada por um aluno de pósgraduação (nível doutorado, com experiência em GF), auxiliando em todos os momentos do seu desenvolvimento, porém, sem interferir na moderação.

\subsection{Competências Gerenciais}

A partir da realização dos $\mathrm{GF}$ (s) foi possível identificar competências gerenciais prioritárias para o ensino do discente de enfermagem como: Tomada de decisão gerencial; Liderança; Relacionamento Interpessoal e Comunicação.

\section{Discussão}

Um dos desafios vivenciados pelas pesquisadoras ao aplicar a técnica de grupo focal foi a dificuldade encontrada na literatura sobre pesquisas que tivessem publicado um guia norteador para o desenvolvimento desta técnica.

Ademais, é relevante destacar que há dificuldade para a elaboração de um guia que responda especificadamente a determinada questão norteadora, ou seja, capaz de atingir o objetivo dos investigadores.

A princípio observou-se que realizar esta técnica de GF seria ideal pelo baixo custo e pela possibilidade de aprofundar discussões, entretanto, vale ressaltar que houve temor se iríamos conseguir concretizar o GF após a construção, pois exige disponibilidade e compreensão no objetivo da técnica que seria aplicada.

A utilização desta técnica auxilia como consulta em etapas de uma pesquisa exploratória qualitativa, quando se busca ampliar a compreensão e a avaliação a cerca de um projeto, programa ou serviço (Ressel et al., 2008), como o caso desta investigação que utilizou esta técnica.

O foco da construção de um guia norteador foi para auxiliar na identificação do objeto do estudo, no caso, as competências gerenciais prioritárias ao desenvolvimento de discentes de enfermagem, contribuindo para a precisão na coleta das informações e condução dos grupos.

A partir dessa premissa, a elaboração de um guia dá a oportunidade ao pesquisador de envolver os participantes da pesquisa, a fim de gerar uma contextualização acerca de uma temática, bem como a reflexão e análise sobre o que se procura investigar, por meio de um processo interativo entre os participantes que gera diferentes opiniões, considerações e sugestões, oriundas das discussões que emergem das questões norteadoras (Soares et al., 2016).

A construção de um guia deve seguir os preceitos ou temas relacionados a área que se almeja pesquisar, sempre em confronto com a literatura disponível e com a validação de especialistas. Nessa linha, pesquisadores evidenciaram diferentes formas de condução do GF na área de moléstias infecciosas com crianças e adolescentes, dos quais avaliaram aspectos positivos (Kinalski et al., 2017). 
Ademais, um exemplo de roteiro apresentado em Gui (2003) estruturado, contém, ao lado de cada tópico, o tempo estimado para tratá-lo (dez minutos, cinco minutos, 15 minutos, etc.). Este não é um procedimento padrão na condução de GF em pesquisa, mas pode auxiliar o pesquisador pouco ambientado ao trabalho com grupos. A maioria dos autores propõe um roteiro curto, focalizado e de fácil uso (Barbour, 2009).

Assim, a construção do guia pode servir de base para condução de demais $G F(s)$ relacionados a área de ensino e pesquisa em enfermagem. Ainda, ressalta-se que nessa experiência, uma vez que os objetivos foram alcançados com os participantes dos grupos, não houve necessidade de utilizar técnicas de apoio, como entrevistas. Esses grupos proporcionaram momentos únicos para o desenvolvimento profissional dos pesquisadores e professores, pois as informações coletadas estabeleceram as bases para uma base qualitativa relacionada ao tema em questão.

Corroborando com este fato, para as sessões grupais não é necessário estabelecer uma quantidade exata de reuniões, ou seja, vai depender se os participantes atingiram o assunto em pauta (Aschidamini \& Saupe, 2004; Gatti, 2012). Assim, ao identificar que se esgotaram nos depoimentos dos docentes, a descrição das competências, encerrou-se os encontros. Ao atingir-se a fase de saturação iniciou-se a análise dos dados.

É notório enfatizar que durante a discussão em GF, além do roteiro com as questões de investigação que o estudo em pauta visa responder, outros temas podem emergir; assim, o moderador deve oferecer orientações para introduzir o assunto desde que não fuja dos objetivos. Nessa direção, o moderador pode exercer várias funções, como garantir a participação e harmonia, esclarecer ou aprofundar temas específicos, conduzir para o próximo tópico quando não houver mais nada a ser dito, estimular as pessoas mais inibidas, bem como desestimular os mais falantes e finalizar o grupo reiterando os principais pontos discutidos (Gatti, 2012; Soares et al., 2016).

O referencial teórico proposto por Gatti (2012) recomenda que as discussões grupais não ultrapassem de 15 pessoas, entretanto, não há um consenso na literatura sobre a quantidade ideal de pessoas, não obstante, quanto menor a participação de docentes, no caso desta investigação, em torno de 4-5, melhor, para potencializar a fala e troca de experiências de cada um dos participantes.

Com relação a implementação efetiva do GF a partir do guia construído, os docentes observaram que apesar de ser importante o ensino de todas as competências gerenciais, o desenvolvimento da liderança, tomada de decisão, comunicação e relacionamento interpessoal se faz prioritário ao ensino.

A liderança, comunicação e relacionamento interpessoal, são temas que nunca se esgotam durante o período de ensino (Reed et al., 2019), além de que, pela inexperiência do aluno, ou pela falta de padronização e/ou utilização de metodologias problematizadoras durante a formação, o aluno tem dificuldade de desenvolver essas competências e acaba por desenvolvê-las no mercado de trabalho. Ademais, as disciplinas dos cursos de enfermagem devem incluir nos seus programas a aprendizagem da tomada de decisão gerencial, comunicação, liderança e relações humanas (Johansen \& O'brien, 2016).

Outrossim, os centros formadores de graduação devem se preocupar em elaborar metodologias ativas de aprendizagem para os futuros profissionais, assim, a técnica de grupo focal reponde com propriedade às exigências de uma formação adequada aos futuros enfermeiros.

Por fim, destaca-se que a experiência de construir um guia e utilizá-lo na condução de grupos focais teve resultados positivos, ao possibilitar identificar as competências gerenciais que devem ser desenvolvidas na graduação, além se provocar a reflexão crítica dos pesquisadores neste método de investigação.

Esta pesquisa apresenta a limitação de ter sido realizada unicamente em uma IES pública e para uma área específica de ensino na enfermagem, não abarcando a análise de demais profissionais e de outros conteúdos gerais para condução de GF. Assim, ressalta-se a importância da realização de outros estudos de forma a abranger outros participantes. 


\section{Conclusão}

Foi possível disponibilizar um guia norteador para condução de GF voltado para área de ensino em enfermagem gerencial, utilizado para identificar as competências gerenciais que devem ser desenvolvidas ainda na graduação. O GF por sua fundamentação na discursividade e interação pressupõe a construção de conhecimento em espaços de intersubjetividade. Portanto, pressupõe-se que muitas vozes ouvidas no grupo contêm aspectos semelhantes e diferentes, de concepção e desconstrução, inerentes à intersubjetividade. É vital conhecer profundamente seus fundamentos e seu processo de desenvolvimento, pois seu sucesso se dará na medida em que os pesquisadores estiverem adequadamente incorporados na pesquisa elegida.

Ademais, a construção de um guia deve favorecer a condução de técnicas metodológicas como o grupo focal, propiciando a transformação dos sujeitos no que diz respeito ao seu conhecimento sobre alguma temática, neste caso, especificamente as competências gerenciais necessárias a formação do enfermeiro.

\section{Agradecimentos}

À Escola de Enfermagem de Ribeirão Preto-USP e ao Conselho Nacional de Desenvolvimento Científico e Tecnológico.

\section{Referências}

Aschidamini, I. M., \& Saupe, R. (2004). Grupo Focal - estratégia metodológica qualitativa: um ensaio teórico. Cogitare Enfermagem, 9(1), 9-14. http://dx.doi.org/10.5380/ce.v9i1.1700

Barbour, R. (2009). Grupos focais. B.Tec Senac: Artmed.

Braun, V., \& Clarke, V. (2006). Using thematic analysis in psychology. Qualitative Research in Psychology, 3(2), 77-101. http://dx.doi.org/10.1191/1478088706qp063oa

Dall'agnol, C. M., Magalhães, A. M. M., Mano, G. C. M., Olschowsky, A., \& Silva, F. P. (2012). A noção de tarefa nos grupos focais. Revista Gaúcha de Enfermagem, 33(1), 186-90. http://dx.doi.org/10.1590/S1983-14472012000100024

Ferracioli, G. V., Oliveira, R. R., Souza, V. S., Teston, E. F., Varela, P. L. R., \& Costa, M. A. R. (2020). Competências gerenciais na perspectiva de enfermeiros do contexto hospitalar. Revista Enfermagem em Foco, 11(1), 15-20. http://revista.cofen.gov.br/index.php/enfermagem/article/view/2254/696

Gatti, B. A. (2012). Grupo focal na pesquisa em ciências sociais e humanas. Autores-Associados: Liber Livro.

Gui, R. (2003). Grupo focal em pesquisa qualitative aplicada: intersubjetividade e construção de sentido. Psicologia: Organizações \& Trabalho, 3(1), 135-160.

http://pepsic.bvsalud.org/scielo.php?script=sci_arttext\&pid=S1984-66572003000100007

Johansen, M. L., \& O'brien, J. L. (2016). Decision making in nursing practice: A concept analysis. Nursing Forum, 51(1), 40-48. http://dx.doi.org/10.1111/nuf.12119.

Kinalski, D. D. F., Paula, C.C., Padoin, S.M.M., Neves, E.T., Kleinubing, R.E., \& Cortes, L. F. (2017). Grupo focal na pesquisa qualitativa: relato de experiência. Revista Brasileira de Enfermagem, 70(2), 424-429. http://dx.doi.org/10.1590/0034-7167-2016-0091

Reed, B. N., Klutts, A. M., \& Mattingly, T. J. (2019). A Systematic Review of Leadership Definitions, Competencies, and Assessment Methods in Pharmacy Education. American Journal of Pharmaceutical Education, 83(9), 7520. http://dx.doi.org/10.5688/ajpe7520

Ressel, L. B., Gualda, D. M. R., \& Gonzáles, R. M. B. (2008). Grupo focal como estratégia para coletar dados de pesquisa.O uso do grupo focal em pesquisa qualitativa. Texto \& Contexto Enfermagem, 17(4), 77986. http://dx.doi.org/10.1590/S0104-07072008000400021

Santos, A. A., Pedreira, L.C., Freitas, R.A, Gomes, N.P., Menezes, T.M.O., \& Moura, L.V.C. (2019). Grupo focal como técnica de coleta de dados na pesquisa em enfermagem: Um relato de experiencia. Revista Eletrônica Acervo Saúde, 11(17), e1648. https://doi.org/10.25248/reas.e1648.2019

Soares, M. I., Camelo, S. H. H., \& Resck, Z. M. R. (2016). Technique of focus group in qualitative data collection: experience report. Revista Mineira de Enfermagem, 20, 1-5. http://www.dx.doi.org/10.5935/1415-2762.20160012 